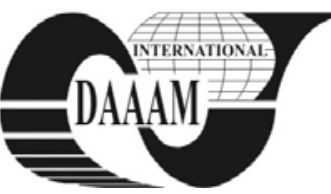

\title{
CALCULUS OF STIRLING ENGINES PERFORMANCES USING THE FIRST LAW FOR FINITE SPEED PROCESSES
}

\author{
FLOREA, T[raian]; PRUIU, A[nastase]; BEJAN, M[ihai]; SERBANESCU, I[on]; CATANA, D[umitru] \& \\ VASILE, F[lorian]
}

\begin{abstract}
This paper presents a technique for calculating the efficiency and power of Stirling engines. This technique is based on the first law of thermodynamics for processes with finite speed. It is presented a new PV/Px diagram that reveals the effects of pressure losses produced by friction, finite speed and throttling processes in the regenerator of the Stirling engine. The results predicted by this analysis are well correlated with the actual engine performance data. The experimental values were collected from twelve different Stirling engines over a large range of output from economy to maximum power. This provides a validation of this theory which is able to accurately predict Stirling engine performances, particular for efficiency and output power.

Key words: finite speed, Stirling, efficiency, power
\end{abstract}

\section{INTRODUCTION}

This paper presents a new technique for calculating the efficiency and power of already existing Stirling engines. It is based on the first law of thermodynamics addressed to finite speed processes and a new method for determining the imperfect regeneration coefficient (Chen \& Yan, 1989), (Florea, 1999).

One of the objectives of this paper is to develop the method for determining the imperfect regeneration coefficient $X$, and to use it for calculating the efficiency and the power output of the Stirling engine.

In the direct Method for the Study and Optimization of Irreversible Cycles with Finite Speed, equations based on the first law of thermodynamics for Processes with finite Speed are integrated in such a manner that the expressions for efficiency and power are obtained directly for any irreversible Cycle. This Method has been applied to Stirling machines previously. This Method has been applied to Stirling machines previously. The analytical results depend upon inclusion of arbitrary coefficients based on experimental data to accurately predict performance. The thermal efficiency is expressed as a product of the Carnot cycle efficiency and second law efficiency

$$
\begin{gathered}
\eta_{S E}=\eta_{C C} \cdot \eta_{I I, \text { irrev }}= \\
\underbrace{\left(1-\frac{T_{L}}{T_{H, S}}\right)}_{\eta_{C C}} \cdot \underbrace{\left[1+\sqrt{\frac{T_{L}}{T_{H, S}}}\right]^{-1}}_{\eta_{I I, \text { irrev }, \Delta T}} \cdot \underbrace{\left[1+\frac{X\left(1-\sqrt{\frac{T_{L}}{T_{H, S}}}\right)}{(\gamma-1) \ln \varepsilon}\right]^{-1}}_{\eta_{I I, \text { irrev }, X}} . \\
\underbrace{\left[1-\frac{3 \mu \sum \Delta P_{i}}{T^{\prime} P_{1} \sqrt{\frac{T_{H, S}}{T_{L}} \ln \varepsilon}}\right]}_{\eta_{I I, \text { irrev }, \Sigma \Delta P_{i}}}
\end{gathered}
$$

with

$$
\begin{gathered}
\eta^{\prime}=\left(1-\sqrt{\frac{T_{L}}{T_{H, S}}}\right) \cdot \eta_{I I, \text { irrev }, X} \\
\mu=1-1 /(3 \varepsilon)
\end{gathered}
$$

The above equations may be used to clearly show the effect of each of the cited losses on the overall efficiency of the engine. The efficiency can be calculated for any speed. The pressure losses and their effect on efficiency and power of the engine depend on the piston speed and the speed of the engine.

The power output of the engine is

$$
\text { Power }_{S E}=\eta_{S E} \cdot m R T_{H, g} \cdot \ln \varepsilon \cdot(w / 2 S)
$$

where $\varepsilon$ is the compression ratio, $w$ is piston speed, $S$ is the stroke of the piston, and $\gamma$ is the specific heat ratio.

The speed for maximum power can be determined since the power output is also a function of the engine speed. Therefore the operating speed of a particular Stirling engine can be selected either for maximum economy or for maximum power. Also, knowledge of the nature of these losses can be effectively used in engine design.

A major cause of losses in Stirling engines is incomplete regeneration. This is revealed by the coefficient of regenerative losses, $X$ (Petrescu, S at al., 2000).

The coefficient of regenerative losses, $X$, is the term that includes all of the losses due to incomplete heat transfer in the regenerator.

This parameter clearly depends on a large number of variables. Among these are piston speed w, cylinder dimensions (diameter $\mathrm{D}_{\mathrm{C}}$ and stroke $\mathrm{S}$ ), regenerator dimensions (diameter $D_{R}$ and length $L$ ), material internal to the regenerator properties (density $\rho_{R}$, specific heat $c_{R}$ ) and dimensions of the screens $(d, b)$, gas properties $\left(p_{g}, c_{p}, \gamma, R\right)$ and the range of operating conditions $(\varepsilon, \tau)$. The relationship expressing $X$ as a function of all these parameters has been evaluated using first law considerations and heat transfer principles for both, the regenerator and the gas.

$$
\begin{gathered}
\eta_{I I, \text { irrev }, X}=\left[1+\frac{\left[X_{1} \cdot y+X_{2} \cdot(1-y)\right]\left(1-\sqrt{T_{L} / T_{H, S}}\right)}{R / c_{v}(T) \ln \varepsilon}\right]^{-1} \\
X_{1}=\frac{1+2 M+e^{-B}}{2(1+M)} ; \quad X_{2}=\frac{M+e^{-B}}{(1+M)} \\
M=\frac{m_{g} c_{v, g}}{m_{R} c_{R}} ; B=(1+M) \frac{h A_{R}}{m_{g} c_{v, g}} \cdot \frac{S}{w} \\
h=\frac{0,395\left(4 P_{m} / R T_{L}\right) w_{g}^{0,424} \cdot c_{p}\left(T_{m}\right) \cdot v\left(T_{m}\right)^{0,576}}{(1+\tau)\left[1-\frac{\pi}{4[(b / d)+1]}\right] D_{R}^{0,576} \cdot \operatorname{Pr}^{2 / 3}}
\end{gathered}
$$

where $y$ is one of the adjusting coefficients of the method having a value of 0,72 .

One objective is to realize an accurate analysis of the pressure losses using a $P V / P x$ diagram. It is also presented a technique used to calculate the efficiency and power of Stirling engines.

The results predicted by this analysis are compared with performance data collected from twelve already existing Stirling engines over a large range of operating conditions. 


\section{THE METHOD OF DETERMINING THE PERFORMANCE OF THE STIRLING ENGINE}

Computations of pressure losses, work losses, efficiency and power of the processes revealed by the new $P V / P x$ diagrams are draw using the first law of thermodynamics for processes with finite speed (Florea et al., 2009). The form of the first law which includes the above mentioned conditions is

$$
d U=\delta Q-P_{m, i}\left(1 \pm \frac{a w}{c} \pm \frac{b \cdot \Delta P_{\text {thrott }}}{2 P_{m, i}} \pm \frac{f \cdot \Delta P_{f}}{P_{m, i}}\right) d V
$$

The irreversible work is

$$
\delta W_{\text {irrev }}=P_{m, i}\left(1 \pm \frac{a w}{c} \pm \frac{b \cdot \Delta P_{\text {thrott }}}{2 P_{m, i}} \pm \frac{f \cdot \Delta P_{f}}{P_{m, i}}\right) d V
$$

when applied to processes with finite speed, as diagram $P V / P x$ reveals.

Computing and adding all pressure losses of the Stirling engine cycle presented above, the term $\eta_{I I, \text { irrev }, \Sigma \Delta P_{i}}$ from eq. (1) becomes:

$$
\begin{gathered}
\eta_{I I, \text { irrev }, \Sigma \Delta P_{i}}= \\
1-\frac{\left(\frac{w}{w_{S, L}}\right) \gamma(1+\sqrt{\pi}) \ln \varepsilon}{\tau \eta^{\prime} \ln \varepsilon}-\frac{5\left(\frac{w}{w_{S, L}}\right)^{2} N+\frac{3(0,94+0,045 w) 10^{5}}{4 \rho_{4}}}{\tau \eta^{\prime} \ln \varepsilon}
\end{gathered}
$$

The heat input during the expansion process is also irreversible because the speed is finite. In order to take into consideration this influence, a calibration coefficient $z$ is introduced

$$
Q_{34}=z \cdot m R T_{H, g} \ln \varepsilon
$$

Finally, the real power output of the engine, eq. (4) becomes

$$
\text { Power }_{S E, \text { irrev }}=\eta_{S E} \cdot z m R T_{H, g} \cdot \ln \varepsilon \cdot(w / 2 S)
$$

where the value of $z$ was evaluated at 0,8 by comparison with experimental data from twelve Stirling engines.

\section{DISCUSSIONS}

The values of the regenerative losses coefficient, $X$, depending by the piston speed for different average gas pressures is revealed by figure 1 (Florea et al., 2006).

Figure 2 shows an accurate predicting of the Stirling engine performance. A comparison of the analysis results with actual performance data for the NS-30S Stirling engine.

The ability to accurately predict the performance of a particular Stirling engine over a large range of operating speeds it is highly desirable in the design of the engine.

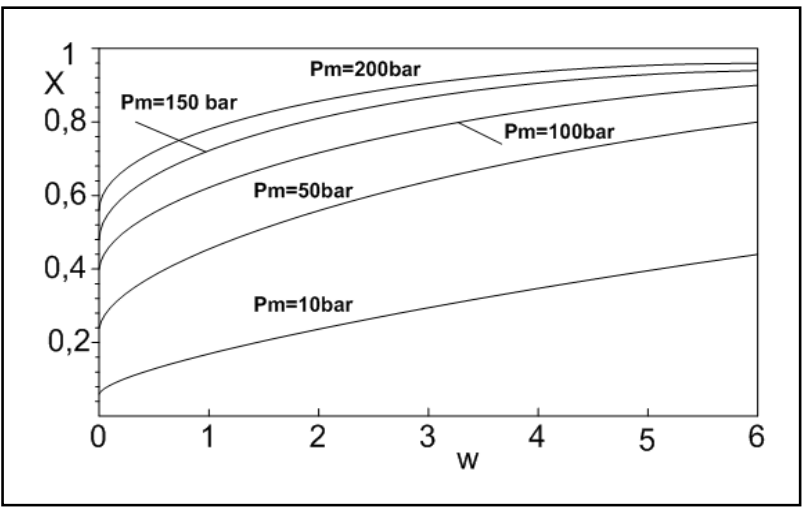

Fig. 1. Coefficient of regenerative losses versus piston speed

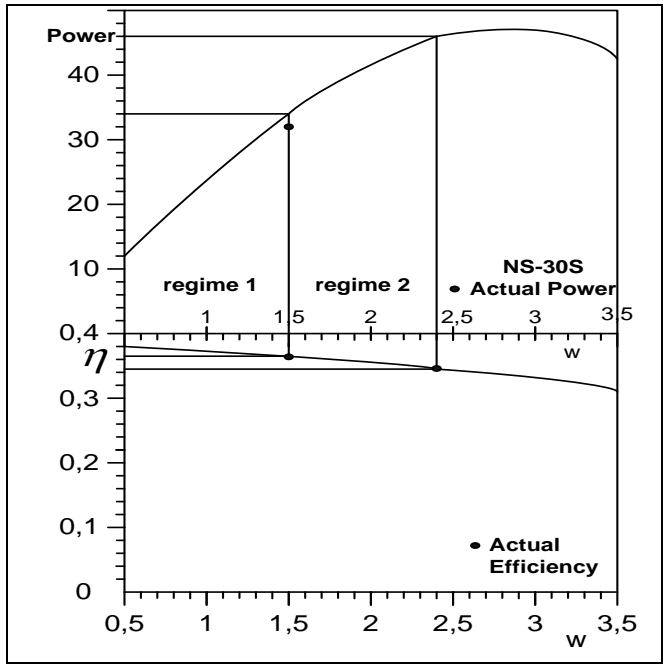

Fig. 2. Theoretical versus experimental performance

\section{CONCLUSION}

The objective of this study was to create analytical models of the already existing Stirling engines. The accuracy of the results was a goal, without losing insight to the mechanisms in order to generate the irreversibilities. Pressure and work losses generated by finite speed of the actual processes were computed as well as the power and efficiency of engines. The first law of thermodynamics for processes with finite speed was used to compute the power losses generated by the pressure losses. The analysis presented was applied to specific operating Stirling cycle engines and results were compared to the actual performance of the engines, experimentally determined. The strong correlation between analytical results and actual engine performance data indicates that the Direct Method of using the First Law for Finite Speed is a valid method of analysis for irreversible cycles.

\section{REFERENCES}

Chen, L. \& Yan, Z. (1989). The Effect of Heat Transfer Law on Performance of a Two-Heat Source Endoreversible Cycle, Journal of Chemical Physics, Vol. 90, pp. 120-126, ISSN 0021-9606

Florea, T. (1999). Grapho-Analytical Method for the Study of Irreversible Processes in Stirling Engines, Ph.D. Thesis, Polytechnic University of Bucharest

Florea, T.; Dragalina, A.; Costiniuc, C.; Florea, E. \& Florea, T.V. (2006). A Method for Calculating of the Coefficient for the Regenerative Losses in Stirling Machines, Acta Technica Napocensis, Series: Aplplied Mathematics and Mechanics, Vol. III, No. 49, May 2006, pp. 747-754, ISBN 1221-5872

Florea, T.; Dragalina, A.; Florea, T. V.; Bejan, M. \& Pruiu, A. (2009). Calculus of Regenerative Losses Coefficient in Stirling Engines 0135-0137, Annals of DAAAM for 2009 \& Proceedings of the $20^{\text {th }}$ International DAAAM Symposium, ISBN 978-3-901509-70-4, ISSN 1726-9679, pp 068, Editor B[ranko] Katalinic, Published by DAAAM International, Vienna

Petrescu, S.; Harman, C.; Florea, T. \& Costea, M. (2000). A Method for Calculating the Coefficient for the Regenerative Losses in Stirling Machines, Proceedings of $5^{\text {th }}$ European Stirling Forum 2000, Ösnabruck 